\title{
Reevaluation of technical protocols and other ways to reduce the radiation dose in computed tomography
}

\author{
Reavaliação de protocolos técnicos e outras maneiras de redução da dose de radiação em tomografia computadorizada
}

$\overline{\text { Jorge Elias Jr. }}{ }^{1}$

The concerns about the radiation dose in computed tomography $(\mathrm{CT})$ have been the object of various review articles as well as research investigations aimed at reducing radiation to the lowest possible levels. This can be exemplified by a survey in the PubMed database (http://www.ncbi.nlm.nih.gov/pubmed) by entering the terms "computerized tomography radiation dose risk", with 247 articles being found in 2012 as compared with only 7 articles found in 1992. As previously mentioned by several authors as well as by a recent editorial in this journal ${ }^{(1)}, \mathrm{CT}$ is the main imaging method related to the increase of general medical radiation, particularly because of the swiftness in its performance, availability and increase in clinical indications, not to mention the augmentation of multislice capability in the latest CT equipments.

Ultimately, there are three main ways that contribute to reduce the radiation generated by $\mathrm{CT}^{(2,3)}$ : a) whenever indicated, substitution of CT either by ultrasonography (US) or magnetic resonance imaging (MRI); b) use CT scans only with well established indications; c) optimization of CT scans parameters aimed to reduce the radiation dose provided this does not imply a loss in diagnostic capability (ALARA principle).

In the present issue of Radiologia Brasileira, Costa et al.(4) present an interesting research article whose results demonstrate that non-contrast-enhanced CT images acquired previously to the dynamic contrast injection, does not significantly contribute for the final diagnosis in most cases with indication for abdominal evaluation $^{(4)}$ and, therefore, could be suppressed. In a previous issue of Radiologia Brasileira, another equally interesting article written by the same group of researchers demonstrated that the equilibrium phase also adds little value to abdominal CT studies for certain indications such as tumor staging, acute abdomen and investigation of abdominal collections ${ }^{(5)}$ and therefore it would be expendable. Those two articles exemplify a relevant aspect to be

1. Professor of Radiology, Center of Imaging Sciences and Medical Physics, Department of Internal Medicine, Ribeirāo Preto School of Medicine, University of Sāo Paulo, Ribeirão Preto, SP, Brazil. E-mail: jejunior@fmrp.usp.br. considered for radiation reduction in $\mathrm{CT}$ scans and that can be classified as optimization of CT parameters. However, it is necessary to bear in mind that the development of specific CT protocols must take into consideration the various possible indications for the exam and, surely, the greater the amount of clinical and laboratory data available, the more appropriate the protocol will be to respond to the CT scan request In this regard, both studies were correct in the subjects'sampling, as both considered the routine activity for the main indications in a hospital setting, which permitted to build consistent results regarding the usefulness of the non-contrast-enhanced and equilibrium phases.

Also, considering the protocols for abdominal CT scans, it is important to highlight that there are evidences supporting the performance of the non-contrast-enhanced phase only in cases of acute abdomen, such as in suspected acute appendicitis, urinary lithiasis and diverticulitis ${ }^{(6)}$, as well as in some clinical situations of acute pancreatitis ${ }^{(7)}$. Even so, radiation dose may represent an issue in cases of excessive use of CT scans, even with non-contrast-enhanced phase only CTs, like in cases of multiple follow-up of urinary lithiasis ${ }^{(8)}$.

Regarding the use of CT examination only with well established indications, it is important to observe that there is an inherent difficulty in the practice of radiology to argue about indications for exams. This is a delicate matter, sometimes involving the financial survival of the service itself, as well as the professional relationship with the requesting physicians. It is very important that all decisions and actions regarding this matter be dealt with under the ethical guiding principles of the medical profession, always placing the patient above all other matters.

Still on this question, the American College of Radiology standardizes the best practices in the utilization of the different imaging methods by means of the "ACR Appropriateness Criteria", which comprises a list of the main clinical situations with the most appropriate imaging methods according to evidence-based data ${ }^{(9)}$. Such a publication reflects a continuous effort, with frequent review of guidelines developed by expert panels, under the light of 
new evidences. It is obvious that, albeit apparently, it is easier to discuss each clinical case in a hospital setting, where it is also easier to institute mechanisms linked to hospital and radiological information systems (HIS and RIS) to guide and educate the requesting colleagues in different medical areas. Under this particular topic, considerable efforts have been undertaken to develop clinical decision support systems in the field of radiology [Computerized Decision Support (CDS) Systems], which analyze the patients' clinical data correlating them with evidence-based databases, allowing the physician to recommend the most appropriate actions for each case, including specific imaging methods recommendations or even precluding any imaging study ${ }^{(10)}$. The enhancement of the quality and safety of radiological services, as well as cost reduction are the main benefits from such a strategy. In those cases where such resources are not available, it is extremely important that we take the role of the patient's advocate assuring the best possible assistance, even in cases where that means delaying, or even reconsidering the performance of a CT scan, in order to discuss with the requesting colleague the possibility of substitution of such a method by another, either US or MRI, depending upon the best indication ${ }^{(11)}$. This is particularly important for children and women in childbearing age, as those two patient groups are the most susceptible to the effects of ionizing radiations ${ }^{(2,3)}$.

In conclusion, there are several ways to reduce ionizing radiation in CT scans, both during the images acquisition as well as by means of clinical discussions, which serve, not only for the case under scrutiny, but also other similar cases. It is extremely important that radiologists be always aware of their role in the guidance about the best practices in imaging diagnosis, by assisting and updating the requesting colleagues, and always providing what is more appropriate for each patient. For such an endeavor, it is fundamental that all colleagues keep themselves constantly updated.

\section{REFERENCES}

1. Parente DB. Radiation risk in the indiscriminate use of CT scan. Radiol Bras 2013;46(2):v-vi.

2. Semelka RC, Armao DM, Elias J Jr, et al. Imaging strategies to reduce the risk of radiation in $\mathrm{CT}$ studies, including selective substitution with MRI. J Magn Reson Imaging. 2007;25:900-9.

3. Armao D, Semelka RC, Elias J Jr. Radiology's ethical responsibility for healthcare reform: tempering the overutilization of medical imaging and trimming down a heavyweight. J Magn Reson Imaging. 2012;35:512-7.

4. Costa DMC, Salvadori PS, Monjardim RF, et al. When the non-contrast-enhanced phase is unnecessary in abdominal computed tomography scans? A retrospective analysis of 244 cases. Radiol Bras. 2013:46:197-202

5. Salvadori PS, Costa DMC, Romano RFT, et al. What is the real role of the equilibrium phase in abdominal computed tomography? Radiol Bras 2013;46:65-70

6. Freire Filho EO, Jesus PEM, D'Ippolito G, et al. When and why use unenhanced computed tomography in patients with acute abdomen. Radiol Bras. 2006:39:51-62

7. Elias J Jr. Utilização da tomografia computadorizada sem contraste e da ressonância magnética no diagnóstico e na estratificação da gravidade da pancreatite aguda. [Tese de Doutorado]. Ribeirāo Preto: Faculdade de Medicina de Ribeirão Preto - Universidade de São Paulo; 2002.

8. Poletti PA, Platon A, Rutschmann OT, et al. Abdominal plain film in patients admitted with clinical suspicion of renal colic: should it be replaced by lowdose computed tomography? Urology. 2006:67:64-8.

9. American College of Radiology. ACR Appropriateness Criteria®). [cited 2013 July 11]. Available from: http://www.acr.org/Quality-Safety/AppropriatenessCriteria.

10. Khorasani R. Computerized physician order entry and decision support: improving the quality of care. Radiographics. 2001;21:1015-8.

11. Amis ES Jr, Butler PF, Applegate KE, et al. American College of Radiology white paper on radiation dose in medicine. J Am Coll Radiol. 2007;4:272-84. 